\title{
Quantitative Relaxometry using Synthetic MRI Could be Better than Qualitative T2-FLAIR Mismatch Sign: A Pilot Study
}

Kazufumi Kikuchi

Kyushu University: Kyushu Daigaku

Akio Hiwatashi (D hiwatasi@radiol.med.kyushu-u.ac.jp)

Kyushu University https://orcid.org/0000-0001-5400-5083

Osamu Togao

Kyushu University: Kyushu Daigaku

Koji Yamashita

Kyushu University: Kyushu Daigaku

Daichi Momosaka

Kyushu University: Kyushu Daigaku

Yoshitomo Kikuchi

Kyushu University: Kyushu Daigaku

Daisuke Kuga

Kyushu University: Kyushu Daigaku

Nobuhiro Hata

Kyushu University: Kyushu Daigaku

Masahiro Mizoguchi

Kyushu University: Kyushu Daigaku

Hidetaka Yamamoto

Kyushu University: Kyushu Daigaku

Toru Iwaki

Kyushu University: Kyushu Daigaku

Kousei Ishigami

Kyushu University: Kyushu Daigaku

\section{Research Article}

Keywords: Synthetic MRI, T2-FLAIR mismatch sign, IDH-mutant, lower-grade glioma

Posted Date: July 6th, 2021

DOI: https://doi.org/10.21203/rs.3.rs-659829/v1 
License: (c) (i) This work is licensed under a Creative Commons Attribution 4.0 International License. Read Full License 


\section{Abstract}

Introduction The diagnostic utility of synthetic MRI (SyMRI) in patients with isocitrate dehydrogenase (IDH)-mutant lower-grade gliomas has not been investigated. The purpose of this study was to determine whether quantitative relaxometry using SyMRI could differentiate between lower-grade gliomas with an increased sensitivity when compared to the qualitative T2-FLAIR mismatch sign.

Methods Between May 2019 and May 2020, thirteen patients with IDH-mutant diffuse gliomas including seven astrocytomas and six oligodendrogliomas were evaluated. Five neuroradiologists independently evaluated the presence of the T2-FLAIR mismatch sign qualitatively. Inter-rater agreement of T2-FLAIR mismatch sign was calculated using the Fleiss kappa coefficient. SyMRI parameters (T1, T2-relaxation times, proton density [PD]) were measured in the gliomas and compared by Mann-Whitney U-test. The receiver operating characteristic analysis was used to evaluate the diagnostic performance. The simulated T2-FLAIR mismatch sign (\%change) was also evaluated.

Results The sensitivity, specificity, and kappa coefficient were $57.1 \%, 83.3 \%$, and 0.38 , respectively in T2FLAIR mismatch sign. There were differences between the gliomas in the $10-90^{\text {th }}$ percentiles and the mean in T1, T2-relaxation times, and PD (all $\mathrm{p}<.05$ ). The T2-relaxation time of the $10^{\text {th }}$ and $50^{\text {th }}$ percentiles, and the mean showed the highest diagnostic performance (area under the curve $=1.00$ ). The $\%$ change of present/absent T2-FLAIR mismatch cases were $-49.3 \pm 6.5 \%,-43.4 \pm 7.9 \%$, respectively.

Conclusion Quantitative relaxometry using SyMRI could differentiate astrocytomas from oligodendrogliomas with an increased sensitivity and objectivity compared to the qualitative T2-FLAIR mismatch sign.

\section{Introduction}

Isocitrate dehydrogenase (IDH) enzymes play a key role in tumorigenesis of glioma. IDH mutations (IDHmutant), including IDH1 and IDH2, were originally identified in glioblastoma [1]. A previous study has revealed that IDH mutations were more frequently observed in diffuse lower-grade gliomas including both astrocytomas and oligodendrogliomas [2]. In lower-grade gliomas, this mutation is an early event in tumor development, which is associated with malignant transformation involving a secondary glioblastoma [3]. Diffuse/anaplastic astrocytomas, IDH-mutant, oligodendroglioma/anaplastic oligodendrogliomas, IDH-mutant and deletion of the short arm of chromosome 1 and long arm of chromosome 19 (1p/19q-codeleted) are considered as lower-grade gliomas [4]. These two types of glioma have the same mutant status as IDH; however, their prognoses vary [5]. Oligodendrogliomas, IDHmutant, and 1p/19q-codeleted are associated with better prognoses [6], and respond better to chemotherapy or radiotherapy [7]. Thus, differential diagnosis is crucial for patient management.

In the differential diagnosis between these lower-grade gliomas, so-called "T2-FLAIR mismatch sign", was reported as a specific imaging marker for IDH-mutant astrocytomas [5]. This highly specific imaging 
marker sign on conventional MRI has the specificity of 100\%; however, it has low sensitivity (51\%) [8], and is considered subjective since its diagnosis is based on the appearance in the imaging modality.

We hypothesized that quantitative relaxometry would increase the sensitivity and objectivity compared to the qualitative T2-FLAIR mismatch sign. Synthetic MRI (SyMRI) can directly evaluate T1- and T2relaxation times, and proton density (PD) values in the clinical scan time and hence was considered for quantitative relaxometry [9]. To the best of our knowledge, no study has quantitatively evaluated the diagnostic accuracy of relaxometry in patients with IDH-mutant lower-grade gliomas.

Hence, the purpose of this study was to determine whether quantitative relaxometry using SyMRI could differentiate between these lower-grade gliomas leading to an increased sensitivity when compared to the qualitative T2-FLAIR mismatch sign.

\section{Material And Methods}

This retrospective, single institution study was approved by our Institutional Review Board, and requirement for informed consent was waived.

\section{Patients}

All patients consecutively diagnosed with glioma at our institution from May 2019 to May 2020 were eligible for this study. Inclusion criteria were 1) patients with glioma, IDH-mutant and/or 1p/19q-codeleted based on the World Health Organization (WHO) classification [4]; 2) MRI scans that had been performed within two weeks prior to surgery; and 3) patients for whom SyMRI was acquired. Exclusion criterion was image distortion such as motion artifacts or noise.

\section{Histopathologic Analysis}

All tissue samples were analyzed based on the WHO 2016 classification [10]. Immunohistochemistry for IDH1 R132H, ATRX, p53, and Ki67 were routinely performed. The IDH1/2 was analyzed using highresolution melting with DNA extracted from the frozen tissues. The $1 p / 19 q$ codeletions were evaluated using a microsatellite-based loss of heterozygosity analysis, with 18 markers, to detect loss of the entire chromosome arm [11].

\section{MRI}

MRI was performed using a 3T MR scanner (Ingenia 3.0T CX; Philips Healthcare, Best, Netherlands) with a 15-channel head coil. Quantitative MRI was performed using the two-dimensional axial quantification of relaxation times and proton density by multi-echo acquisition of a saturation-recovery using turbo spinecho readout (QRAPMASTER) pulse sequence with two echo times (TEs; 13 and $100 \mathrm{~ms}$ ) and four delay times to generate eight real images and eight imaginary images [9]. The other parameters included repetition time (TR), $4831 \mathrm{~ms}$; flip angle (FA), 90®; number of excitation (NEX), 1; sensitivity encoding factor, 2.2; field-of-view (FOV), $230 \times 189$ (recon. 230²) mm²; matrix, 512²; echo-train length, 10; 
thickness/gap, 4.0/1.0 mm; 30 slices, and scan time 6 min $36 \mathrm{~s}$. Quantification map acquisition was performed with SyMRI software (Version 19.0; SyMRI, Linköping, Sweden). Standard MR sequences (T1WI, T2WI, FLAIR, and contrast-enhanced T1WI) were also obtained. The sequence parameters of the two-dimensional axial T2WI and FLAIR were as follows: T2Wl; TR/TE, 3000/80 ms; FA, 90区; NEX, 1; FOV, $230^{2} \mathrm{~mm}^{2}$; matrix, $512 \times 375$ (recon. 512²); echo-train length, 15; thickness/gap, 5.0/1.0 mm; 22 slices, and scan time, 2 min 36 s, and FLAIR; TR/TE/TI, 10000/120/2700 ms; FA, 180囚; NEX, 1; FOV, $230 \times 207$ (recon. $230^{2}$ ) mm²; matrix, $320 \times 228$ (recon. $512^{2}$ ); echo-train length, 27; thickness/gap, 5.0/1.0 mm; 22 slices, and scan time, 3 min.

\section{Qualitative evaluation}

Five board-certified neuroradiologists (with 23, 21, 19, 8, and 6 years of experience, respectively) were blinded to the patient information of the evaluated T2-FLAIR mismatch sign $[5,8]$. The T2-FLAIR mismatch sign was defined as follows [5]:

1. Tumor displays complete or near-complete and almost homogenous hyperintense signal on $\mathrm{T} 2 \mathrm{WI}$

2. Tumor displays relatively hypointense signal on the FLAIR except for a hyperintense peripheral rim

They read both the T2WI and FLAIR based on whether the T2-FLAIR mismatch sign was present or absent. Sensitivity, specificity, positive predictive value (PPV), negative predictive value (NPV), and accuracy were calculated.

\section{Quantitative evaluation}

We used a single maximum section of each tumor for the ROI analysis on the T2-prolonged region in the tumor as well as the normal appearing white matter using an ImageJ-plugin (ImageJ/Fiji; version 2.0.0-rc59/1.51k, National Institutes of Health, Bethesda, MD). The ROI was copied and placed on each parameter map $(T 1, T 2, P D)$ to obtain pixel-by-pixel values for the histogram analyses. The 10th, 25th, 50th, 75th, and 90th percentiles, as well as the mean, skewness, and kurtosis, of each parameter were recorded from the histograms.

\section{Simulated T2-FLAIR mismatch sign}

On further investigation, we evaluated the difference in signal intensity $(\mathrm{SI})$ calculated using spin-echo, and inversion recovery in gliomas.

For SI calculation, $\mathrm{SI}_{\text {Spin Echo }}$ and $S I_{\text {Inversion Recovery }}$ were defined by the following equations:

$\mathrm{SI}_{\text {Spin Echo }}=[1-\exp (-\mathrm{TR} / \mathrm{T} 1)] \times \exp (-\mathrm{TE} / \mathrm{T} 2),(1)$

$\mathrm{SI}_{\text {Inversion Recovery }}=[1-2 \times \exp (-\mathrm{Tl} / \mathrm{T} 1)+\exp (-\mathrm{TR} / \mathrm{T} 1)] \times \exp (-\mathrm{TE} / \mathrm{T} 2),(2)$

Here, we applied these values, respectively: TR/TE, 3000/80 ms in SI Spin Echo (for T2WI), TR/TI/TE, 10000/2700/120 ms in SI Inversion Recovery (for FLAIR). Then, the simulated T2-FLAIR mismatch sign (\% 
change) expressed as ratio was defined by the following equation:

Simulated T2-FLAIR mismatch sign (\% change) $=\left(\mathrm{SI}_{\text {Inversion Recovery, FLAIR }}-\mathrm{SI}\right.$ Spin Echo, T2WI $) / \mathrm{SI}_{\text {Spin Echo, }}$ $\mathrm{T} 2 \mathrm{WI} \times 100$ [\%], (3)

\section{Statistical analysis}

Chi-square tests were used for the comparison of the categorical variables in the patients. In the qualitative evaluation, the inter-rater agreement for the T2-FLAIR mismatch sign among five observers was evaluated using the Fleiss's kappa coefficient [12]. The kappa-value was interpreted as follows: almost perfect agreement, 1.00-0.81; substantial agreement, $0.80-0.61$; moderate agreement, $0.60-0.41$; fair agreement, 0.40-0.21; slight agreement, $0.20-0.01$; and poor agreement, $<0$ [13]. In the quantitative evaluation, the percentiles, mean, skewness, and kurtosis of each parameter (i.e., T1, T2-relaxation times, and PD) were compared between astrocytomas and oligodendrogliomas by Mann-Whitney $U$ test. The diagnostic performance of each parameter was evaluated by receiver operating characteristic (ROC) analysis. Comparison of simulated T2-FLAIR mismatch sign was tested by Mann-Whitney Utest. All statistical analyses were performed using commercial software programs (JMP, version 15.0.0; SAS Institute, Cary, NC, USA; Prism 7.0, GraphPad Software, La Jolla, CA, USA). $P$ values $<0.05$ were considered statistically significant.

\section{Results}

\section{Patient Demographics and Characteristics}

A total of 13 patients (median age, 43 years; interquartile range, 36.8-51.2 years; six male and seven female patients) were included in this study. There were seven patients with astrocytoma, IDH-mutant (range, median age: 29-49, 41 years; three males and four females), and six with oligodendroglioma, IDH-mutant and 1p/19q-codeleted (37-63, median age: 48 years; three males and three females). An overview of the patient demographics and characteristics are presented in Table 1, and the case selection process is depicted in Fig. 1. There was a significant difference observed in the T2-FLAIR mismatch sign between the gliomas $(p=0.02)$. The patients' demographics and pathological diagnoses, based on the 2016 WHO classification[4] were as follows: seven astrocytomas included five diffuse astrocytomas (WHO grade II; age, 33-49 years; two males and three females), and two anaplastic astrocytomas (WHO grade III; age, 29 and 45 years; one male and one female). Six oligodendrogliomas included four oligodendrogliomas (WHO grade Il; age, 40-63 years; two males and two females), and two anaplastic oligodendrogliomas (WHO grade III; age, 37 and 43 years; one male and one female). All the patients were genetically verified as IDH-mutant type. Postoperative recurrent cases (three astrocytomas and two oligodendrogliomas) were included. 
Table 1

Patient Demographics and Characteristics

\begin{tabular}{|c|c|c|c|c|}
\hline Parameter & $\begin{array}{l}\text { Astrocytic } \\
\text { tumor } \\
\text { IDH-mutant }\end{array}$ & $\begin{array}{l}\text { Oligodendroglial tumor } \\
\text { IDH-mutant and } 1 \mathrm{p} / 19 \mathrm{q}- \\
\text { codeleted }\end{array}$ & All gliomas & $\begin{array}{l}{ }^{a} p- \\
\text { value }\end{array}$ \\
\hline No. of patients & 7 & 6 & 13 & $\ldots$ \\
\hline \multirow[t]{2}{*}{ Median age (y) } & $41(29-49)$ & $48(37-63)$ & $43(29-63)$ & \multirow[t]{2}{*}{0.07} \\
\hline & {$[33.8,45.8]$} & {$[39.8,58.4]$} & {$[36.8,51.2]$} & \\
\hline Sex $(M / W)$ & $3 / 4$ & $3 / 3$ & $6 / 7$ & 0.79 \\
\hline WHO Grade (II/III/IV) & $4 / 1 / 2$ & $4 / 2 / 0$ & $8 / 3 / 2$ & 0.32 \\
\hline \multirow[t]{2}{*}{ Tumor size $\left[\mathrm{mm}^{2}\right]$} & $\begin{array}{l}808(356- \\
2379)\end{array}$ & $1477(366-4640)$ & $\begin{array}{l}1380(356- \\
4640)\end{array}$ & \multirow[t]{2}{*}{0.27} \\
\hline & {$[493,1746]$} & {$[911,2482]$} & {$[510,1755]$} & \\
\hline Location (F/P/I) & $4 / 1 / 2$ & $5 / 0 / 1$ & $9 / 1 / 3$ & 0.50 \\
\hline Enhancement (+/-) & $1 / 6$ & $2 / 4$ & $3 / 10$ & 0.42 \\
\hline Calcification (+/-) & $0 / 7$ & $1 / 5$ & $1 / 12$ & 0.26 \\
\hline Cystic component (+/-) & $2 / 5$ & $2 / 4$ & $4 / 9$ & 0.85 \\
\hline Hemorrhage (+/-) & $2 / 5$ & $2 / 4$ & $4 / 9$ & 0.85 \\
\hline $\begin{array}{l}\text { T2-FLAIR mismatch sign } \\
(+/-)\end{array}$ & $4 / 3$ & $0 / 6$ & $4 / 9$ & 0.03 \\
\hline \multicolumn{5}{|c|}{ Data in parentheses are range and data in brackets are interquartile range. } \\
\hline \multicolumn{5}{|c|}{$\begin{array}{l}1 p / 19 q \text {-codeleted }=\text { deletion of the short arm of chromosome } 1 \text { and long arm of chromosome } 19, F= \\
\text { frontal lobe, } I=\text { insula, } I D H=\text { isocitrate dehydrogenase, } M=\text { man, } P=\text { parietal lobe, } W=\text { woman, } W H O= \\
\text { World Health Organization }\end{array}$} \\
\hline
\end{tabular}

\section{Qualitative evaluation}

Table 2 shows the qualitative results by the five neuroradiologists. Averaged sensitivity, specificity, accuracy, PPV, and NPV were $57.1 \%, 83.3 \%, 69.2 \%, 83.6 \%$, and $62.6 \%$, respectively. A fair inter-rater agreement was observed (kappa value $=0.38$ ). 
Table 2

Qualitative evaluation based on T2-FLAIR mismatch sign among 5-radiologists

\begin{tabular}{|c|c|c|c|c|c|c|}
\hline & Reader 1 & Reader 2 & Reader 3 & Reader 4 & Reader 5 & Average \\
\hline \multirow{2}{*}{$\begin{array}{l}\text { Sensitivity } \\
{[\%]}\end{array}$} & $57.1(4 / 7)$ & $42.9(3 / 7)$ & $57.1(4 / 7)$ & $57.1(4 / 7)$ & $71.4(5 / 7)$ & 57.1 \\
\hline & $\begin{array}{l}{[18.4} \\
90.1]\end{array}$ & {$[9.9,81.6]$} & {$[18.4,90.1]$} & $\begin{array}{l}{[18.4} \\
90.1]\end{array}$ & $\begin{array}{l}{[29.0} \\
96.3]\end{array}$ & \\
\hline \multirow{2}{*}{$\begin{array}{l}\text { Specificity } \\
{[\%]}\end{array}$} & $66.7(4 / 6)$ & $100.0(6 / 6)$ & $100.0(6 / 6)$ & $83.3(5 / 6)$ & $66.7(4 / 6)$ & 83.3 \\
\hline & $\begin{array}{l}{[22.3} \\
95.7]\end{array}$ & {$[54.1,100]$} & {$[54.1,100]$} & $\begin{array}{l}{[35.9,} \\
99.6]\end{array}$ & $\begin{array}{l}{[22.3} \\
95.7]\end{array}$ & \\
\hline \multirow[t]{2}{*}{ Accuracy [\%] } & 61.5 & 69.2 & 76.9 & 69.2 & 69.2 & 69.2 \\
\hline & $\begin{array}{l}{[31.6,} \\
86.1]\end{array}$ & {$[38.6,90.9]$} & {$[46.2,95.0]$} & $\begin{array}{l}\text { [38.6, } \\
90.9]\end{array}$ & $\begin{array}{l}{[38.6,} \\
90.9]\end{array}$ & \\
\hline \multirow[t]{2}{*}{ PPV [\%] } & 66.7 & 100.0 & 100.0 & 80.0 & 71.4 & 83.6 \\
\hline & $\begin{array}{l}\text { [35.3, } \\
88.0]\end{array}$ & $\begin{array}{l}\text { [not } \\
\text { applicable] }\end{array}$ & $\begin{array}{l}\text { [not } \\
\text { applicable] }\end{array}$ & $\begin{array}{l}{[37.4,} \\
96.4]\end{array}$ & $\begin{array}{l}{[42.4,} \\
89.5]\end{array}$ & \\
\hline \multirow[t]{2}{*}{ NPV [\%] } & 57.1 & 60.0 & 66.7 & 62.5 & 66.7 & 62.6 \\
\hline & $\begin{array}{l}{[32.4,} \\
78.8]\end{array}$ & {$[44.1,74.0]$} & {$[46.0,82.5]$} & $\begin{array}{l}\text { [39.7, } \\
80.8]\end{array}$ & $\begin{array}{l}{[35.3,} \\
88.0]\end{array}$ & \\
\hline$N P V$, negative & redictive va & $P P V$, posit & edictive value & & & \\
\hline
\end{tabular}

\section{Quantitative evaluation}

Figure 2 and Supplemental Material 1 show the histograms of each parameter over all the pixels in the tumor ROIs. T1-, and T2-relaxation times, and PD from the astrocytomas all exhibited a slight rightward shift relative to those from the oligodendrogliomas. There were significant differences in the 10-90th percentiles and mean in T1, T2-relaxation times, and PD (all $p<0.05$ ). There were no significant differences between the astrocytoma and the oligodendroglioma in normal appearing white matter for all the parameters. Table 3 shows the diagnostic performance in differentiating the two gliomas; the most useful values of each parameter are shown in the Table 3. The entire set of our results is shown in the Supplemental Material 2. The T2-relaxation time of the 10th and 50th percentiles, and the mean showed the highest diagnostic performance (area under the curve $[A U C]=1.00$ ) with an optimal diagnosis cutoff value of $99.5,148.3,178.2 \mathrm{~ms}$, respectively. 
Table 3

Diagnostic performance of parameters in differentiating between astrocytic tumor, IDH-mutant and oligodendroglial tumor, IDH-mutant and 1p/19q-codeleted

\begin{tabular}{|c|c|c|c|c|c|c|c|}
\hline Parameters & $\begin{array}{l}\text { Sensitivity } \\
{[\%]}\end{array}$ & $\begin{array}{l}\text { Specificity } \\
\text { [\%] }\end{array}$ & $\begin{array}{l}\text { Accuracy } \\
{[\%]}\end{array}$ & PPV [\%] & NPV [\%] & Cutoff & AUC \\
\hline \multicolumn{8}{|l|}{ T1 [ms] } \\
\hline \multirow{2}{*}{$\begin{array}{l}\text { 50th } \\
\text { percentile }\end{array}$} & 100.0 & \multirow{2}{*}{$\begin{array}{l}83.3(5 / 6) \\
{[35.9,99.6]}\end{array}$} & \multirow{2}{*}{$\begin{array}{l}92.3 \\
{[64.0,} \\
99.8]\end{array}$} & \multirow{2}{*}{$\begin{array}{l}87.5 \\
{[53.9,97.7]}\end{array}$} & 100.0 & \multirow[t]{2}{*}{1332} & \multirow[t]{2}{*}{0.95} \\
\hline & $\begin{array}{l}{[59.0,} \\
100.0]\end{array}$ & & & & $\begin{array}{l}\text { [not } \\
\text { applicable] }\end{array}$ & & \\
\hline \multirow{2}{*}{$\begin{array}{l}\text { 90th } \\
\text { percentile }\end{array}$} & $85.7(6 / 7)$ & $\begin{array}{l}100.0 \\
(6 / 6)\end{array}$ & \multirow{2}{*}{$\begin{array}{l}92.3 \\
\\
{[64.0,} \\
99.8]\end{array}$} & \multirow{2}{*}{$\begin{array}{l}100.0 \\
\text { [not } \\
\text { applicable] }\end{array}$} & 85.7 & \multirow[t]{2}{*}{2290} & \multirow[t]{2}{*}{0.95} \\
\hline & $\begin{array}{l}\text { [42.1, } \\
99.6]\end{array}$ & $\begin{array}{l}{[54.1} \\
100.0]\end{array}$ & & & {$[49.4,97.4]$} & & \\
\hline \multirow[t]{2}{*}{ Mean } & 100.0 & \multirow{2}{*}{$\begin{array}{l}83.3(5 / 6) \\
{[35.9,99.6]}\end{array}$} & \multirow{2}{*}{$\begin{array}{l}92.3 \\
{[64.0} \\
99.8]\end{array}$} & \multirow{2}{*}{$\begin{array}{l}87.5 \\
{[53.9,97.7]}\end{array}$} & 100.0 & \multirow[t]{2}{*}{1407} & \multirow[t]{2}{*}{0.95} \\
\hline & $\begin{array}{l}{[59.0,} \\
100.0]\end{array}$ & & & & $\begin{array}{l}\text { [not } \\
\text { applicable] }\end{array}$ & & \\
\hline \multicolumn{8}{|l|}{ T2 [ms] } \\
\hline \multirow{2}{*}{$\begin{array}{l}\text { 10th } \\
\text { percentile }\end{array}$} & 100.0 & 100.0 & \multirow{2}{*}{$\begin{array}{l}100.0 \\
{[75.3} \\
100.0]\end{array}$} & \multirow{2}{*}{$\begin{array}{l}100.0 \\
\text { [not } \\
\text { applicable] }\end{array}$} & 100.0 & \multirow[t]{2}{*}{100} & \multirow[t]{2}{*}{1.00} \\
\hline & $\begin{array}{l}{[59.0} \\
100.0]\end{array}$ & $\begin{array}{l}\text { [54.1, } \\
\text { 100.0] }\end{array}$ & & & $\begin{array}{l}\text { [not } \\
\text { applicable] }\end{array}$ & & \\
\hline \multirow{2}{*}{$\begin{array}{l}\text { 50th } \\
\text { percentile }\end{array}$} & 100.0 & 100.0 & \multirow{2}{*}{$\begin{array}{l}100.0 \\
{[75.3} \\
100.0]\end{array}$} & \multirow{2}{*}{$\begin{array}{l}100.0 \\
\text { [not } \\
\text { applicable] }\end{array}$} & 100.0 & \multirow[t]{2}{*}{148} & \multirow[t]{2}{*}{1.00} \\
\hline & $\begin{array}{l}\text { [59.0, } \\
100.0]\end{array}$ & $\begin{array}{l}\text { [54.1, } \\
100.0]\end{array}$ & & & $\begin{array}{l}\text { [not } \\
\text { applicable] }\end{array}$ & & \\
\hline \multirow[t]{2}{*}{ Mean } & $\begin{array}{l}100.0 \\
(7 / 7)\end{array}$ & $\begin{array}{l}100.0 \\
(6 / 6)\end{array}$ & \multirow{2}{*}{$\begin{array}{l}100.0 \\
{[75.3} \\
100.0]\end{array}$} & \multirow{2}{*}{$\begin{array}{l}100.0 \\
\text { [not } \\
\text { applicable] }\end{array}$} & 100.0 & \multirow[t]{2}{*}{178} & \multirow[t]{2}{*}{1.00} \\
\hline & $\begin{array}{l}\text { [59.0, } \\
100.0]\end{array}$ & $\begin{array}{l}{[54.1} \\
100.0]\end{array}$ & & & $\begin{array}{l}\text { [not } \\
\text { applicable] }\end{array}$ & & \\
\hline \multicolumn{8}{|l|}{ PD [\%] } \\
\hline 10th & $71.4(5 / 7)$ & $100.0(6 / 6)$ & 84.6 & 100.0 & 75.0 & 81.8 & 0.90 \\
\hline & $\begin{array}{l}\text { [29.0, } \\
96.3]\end{array}$ & $\begin{array}{l}\text { [54.1, } \\
100.0]\end{array}$ & $\begin{array}{l}\text { [54.6, } \\
98.1]\end{array}$ & $\begin{array}{l}\text { [not } \\
\text { applicable] }\end{array}$ & {$[48.2,90.6]$} & & \\
\hline
\end{tabular}

$A U C$, area under the curve; $I D H$, isocitrate dehydrogenase; $N P V$, negative predictive value; $P D$, proton density; PPV, positive predictive value

Data in parentheses are numerator/denominator; data in brackets are $95 \%$ confidence intervals. 


\begin{tabular}{|c|c|c|c|c|c|c|c|}
\hline Parameters & $\begin{array}{l}\text { Sensitivity } \\
{[\%]}\end{array}$ & $\begin{array}{l}\text { Specificity } \\
{[\%]}\end{array}$ & $\begin{array}{l}\text { Accuracy } \\
{[\%]}\end{array}$ & PPV [\%] & NPV [\%] & Cutoff & AUC \\
\hline \multirow{2}{*}{$\begin{array}{l}\text { 25th } \\
\text { percentile }\end{array}$} & $71.4(5 / 7)$ & $100.0(6 / 6)$ & 84.6 & 100.0 & 75.0 & 84.0 & 0.90 \\
\hline & $\begin{array}{l}\text { [29.0, } \\
96.3]\end{array}$ & $\begin{array}{l}{[54.1,} \\
100.0]\end{array}$ & $\begin{array}{l}\text { [54.6, } \\
98.1]\end{array}$ & $\begin{array}{l}\text { [not } \\
\text { applicable] }\end{array}$ & {$[48.2,90.6]$} & & \\
\hline \multirow{2}{*}{$\begin{array}{l}\text { 50th } \\
\text { percentile }\end{array}$} & $71.4(5 / 7)$ & $100.0(6 / 6)$ & 84.6 & 100.0 & 75.0 & 86.4 & 0.90 \\
\hline & $\begin{array}{l}{[29.0} \\
96.3]\end{array}$ & $\begin{array}{l}{[54.1} \\
100.0\end{array}$ & $\begin{array}{l}{[54.6} \\
98.1]\end{array}$ & $\begin{array}{l}\text { [not } \\
\text { applicable] }\end{array}$ & {$[48.2,90.6]$} & & \\
\hline \multirow[t]{2}{*}{ Mean } & $71.4(5 / 7)$ & $100.0(6 / 6)$ & 84.6 & 100.0 & 75.0 & 86.7 & 0.90 \\
\hline & $\begin{array}{l}{[29.0} \\
96.3]\end{array}$ & $\begin{array}{l}{[54.1} \\
100.0]\end{array}$ & $\begin{array}{l}{[54.6,} \\
98.1]\end{array}$ & $\begin{array}{l}\text { [not } \\
\text { applicable] }\end{array}$ & {$[48.2,90.6]$} & & \\
\hline \multicolumn{8}{|c|}{$\begin{array}{l}A U C \text {, area under the curve; } I D H \text {, isocitrate dehydrogenase; } N P V \text {, negative predictive value; } P D \text {, proton } \\
\text { density; } P P V \text {, positive predictive value }\end{array}$} \\
\hline \multicolumn{8}{|c|}{ Data in parentheses are numerator/denominator; data in brackets are $95 \%$ confidence intervals. } \\
\hline
\end{tabular}

Based on the observer test, four cases with astrocytoma were present (true positive) in the T2-FLAIR mismatch sign. The tumor sizes of true positive, false negative astrocytomas, and oligodendroglioma (mean \pm standard deviation) were $1081 \pm 759.9 \mathrm{~mm}^{2}, 1238 \pm 997.9 \mathrm{~mm}^{2}$, and $1803 \pm 1473.1 \mathrm{~mm}^{2}$, respectively. The \% change of present/absent T2-FLAIR mismatch cases were $-49.3 \pm 6.5 \%,-43.4 \pm$ $7.9 \%$, respectively (Table 4, Fig. 3). There were no significant differences between the present/absent T2FLAIR mismatch sign in size and \% change. 
Table 4

Tumor size and \% change among lower-grade gliomas

\begin{tabular}{|c|c|c|c|c|}
\hline \multirow[t]{3}{*}{$\begin{array}{l}\text { T2-FLAIR } \\
\text { mismatch sign }\end{array}$} & \multirow{2}{*}{$\begin{array}{l}\text { Present } \\
\begin{array}{l}\text { Astrocytoma (True } \\
\text { Positive) }\end{array}\end{array}$} & \multicolumn{2}{|l|}{ Absent } & \multirow[t]{3}{*}{$\begin{array}{l}{ }^{\text {a } P} \\
\text { value }\end{array}$} \\
\hline & & $\begin{array}{l}\text { Astrocytoma (False } \\
\text { Negative) }\end{array}$ & & \\
\hline & $(\mathrm{N}=4)$ & $(\mathrm{N}=3)$ & $(N=6)$ & \\
\hline \multirow[t]{2}{*}{ Size $\left[\mathrm{mm}^{2}\right]$} & $1081 \pm 759.9$ & $1614 \pm 1298$ & & 0.50 \\
\hline & & $1238.096 \pm 997.9$ & $1802.65 \pm 1473.1$ & \\
\hline \multirow[t]{2}{*}{ \%change [\%] } & $-49.3 \pm 6.5$ & $-43.4 \pm 7.9$ & & 0.14 \\
\hline & & $-46.6 \pm 12.8$ & $-42.2 \pm 4.2$ & \\
\hline \multicolumn{5}{|c|}{ Data are expressed as mean \pm standard deviation. } \\
\hline
\end{tabular}

Figures 4 and 5 show the representative images of the patients with astrocytoma and oligodendroglioma, respectively.

\section{Discussion}

As anticipated, our results confirmed that quantitative relaxometry using SyMRI could differentiate the IDH-mutant gliomas leading to an increased sensitivity compared to the qualitative T2-FLAIR mismatch sign. Qualitative results showed high specificity; however, sensitivity was low and inter-rater agreement was fair. Quantitative results showed that there were significant differences in the 10-90th percentiles and the mean between the gliomas for all SyMRI parameters. The ROC analysis revealed that the T2value of the 10th and 50th percentiles, and the mean showed the highest diagnostic ability in differentiating gliomas. In the simulated T2-FLAIR mismatch sign, T2-FLAIR mismatch present cases showed a higher mismatch ratio compared to that of the absent cases.

Previous studies have reported that the T2-FLAIR mismatch sign has been shown to predict astrocytoma, IDH-mutant with 95-100\% specificity [14, 15]; however, sensitivity has been low at $46-51 \%[8,14,16]$. Inter-rater agreement has been shown to have a wide range of $K=0.38-0.88[5,8,14,15]$. Our results are consistent with these previous results. Low sensitivity is probably due to the binary scoring system used, whereas subtle changes may result in different interpretations across readers [8]. Some readers in this study also reported the T2-FLAIR mismatch in oligodendroglioma; In this case, a cystic change was present in the tumor. Since T2-FLAIR mismatch occurs based on the long T1-relaxation time, the intratumoral cystic component simulates a T2-FLAIR mismatch. The T2-FLAIR mismatch false positive results have been reported in previous studies $[15,17]$. Therefore, surgical specimens are still needed to diagnose gliomas instead of relying on the T2-FLAIR mismatch sign alone. 
Our quantitative results showed long T1- and T2-relaxation times and an increased PD within the astrocytoma compared to the oligodendroglioma. To our knowledge, this is the first study to quantitatively evaluate IDH-mutant gliomas using SyMRI. These prolonged T1- and T2-relaxation times imply the result of fluid changes in the IDH-mutant astrocytoma. Deguchi et al. revealed that abundant microcysts were observed upon hematoxylin-eosin staining of specimens from T2-FLAIR mismatched region in the astrocytoma with IDH-mutant [15], which may reflect T1- and T2-relaxation time prolongations. Kinoshita et al. evaluated gliomas quantitatively using MP2RAGE images calculated via Bayesian inference modeling and showed that T2-FLAIR mismatch region exhibited extremely long T1and T2-relaxation times [16]. Their results also support fluid changes within the IDH-mutant astrocytoma. Our quantitative results showed that there were significant differences in the mean value between the gliomas for all SyMRI parameters. These findings may support clinical utility of SyMRI to diagnose gliomas because we require a simple ROI to place in the tumor, not requiring a histogram analysis. Further, as expected, quantitative evaluation significantly increased the sensitivity compared to the qualitative T2-FLAIR mismatch sign. Advanced MR techniques such as dynamic perfusion MRI [18] and magnetic resonance spectroscopy [19] have also revealed differences between the IDH-mutant and wildtype gliomas. However, dynamic perfusion MRI requires contrast media and 2-hydroxyglutarate magnetic resonance spectroscopy have been shown to be associated with false positive cases with intratumoral hemorrhage [20]. In addition, 2-hydroxyglutarate detection with magnetic resonance spectroscopy is a technically challenging due to the spectral overlap of 2-hydroxyglutarate with background metabolites [21].

Our result of the simulated T2-FLAIR mismatch sign (\% change) suggested that radiologists were able to detect the difference between T2WI and FLAIR only when it was approximately $>50 \%$. A previous study reported that astrocytoma, IDH-mutant did not always reveal the T2-FLAIR mismatch sign (only 22\%) [5]. Kinoshita et al. tested that shortening the TI could contribute to better visualization of the T2-FLAIR mismatch signs and suggested that fine-tuning inversion recovery sequence may improve the detection in IDH-mutant gliomas [16]. Our quantitative evaluation using SyMRI could overcome this low-sensitivity and inter-observer variability. Contrary to expectations, the tumor size showed the opposite result owing to the sample size in the simulated study, thereby, resulting in a large variation.

This study has several limitations. First, the sample size was small, and our study included postoperative cases. Although postoperative changes may affect the results of the qualitative evaluation, the quantitative evaluation was still useful to differentiate the gliomas in this study. Second, our study did not evaluate the IDH-wildtype astrocytomas. It would be desirable to proceed with a subsequent study including patients with astrocytoma, IDH-wildtype. Third, we did not include the whole tumor volume in the histogram analysis. Instead, we used the maximum section of the tumor, with its boundary defined by the hyperintensity on T2WI. However, in previous studies on the T2-FLAIR mismatch, the whole-volume histogram analysis has not been performed and only the maximum section of the tumor has been utilized. 


\section{Conclusions}

Relaxometry using SyMRI could differentiate astrocytomas, IDH-mutant from oligodendrogliomas, IDHmutant, and 1p/19q-codeleted. Quantitative relaxometry could lead to an increased sensitivity and objectivity compared to the qualitative T2-FLAIR mismatch sign; therefore, this objective evaluation provides a helpful, noninvasive diagnostic method for differentiating IDH-mutant gliomas.

\section{Declarations}

Author contributions: KK: Conceptualization; Methodology; Software; Validation; Formal analysis; Investigation; Resources; Data Curation; Writing-Original Draft; Visualization; AH: Validation; Investigation; Data Curation; Writing - Review \& Editing; Project administration; OT: Validation; Investigation; Resources; Data Curation; Writing-Review \& Editing; KY: Validation; Investigation; Resources; Data Curation; Writing-Review \& Editing; DM: Validation; Investigation; Resources; Data Curation; Writing-Review \& Editing; YK: Validation; Investigation; Resources; Data Curation; WritingReview \& Editing; DK: Validation; Investigation; Resources; Writing-Review \& Editing; NH: Validation; Investigation; Resources; Writing-Review \& Editing; MM: Validation; Investigation; Resources; WritingReview \& Editing; HY: Validation; Investigation; Resources; Writing-Review \& Editing; TI: Validation; Investigation; Resources; Writing-Review \& Editing; Kl: Conceptualization; Methodology; Validation; Investigation; Resources; Writing-Review \& Editing; Supervision; Project administration.

Funding: This work was supported by the Shin-Nihon Foundation of Advanced Medical Research.

Data availability: The datasets generated during the current study are available from the corresponding author on reasonable request.

\section{Declarations}

Conflict of interest: We declare that we have no conflict of interest.

Ethical approval: All procedures performed in studies involving human participants were in accordance with the ethical standards of the institutional and/or national research committee and with the 1964 Helsinki Declaration and its later amendments or comparable ethical standards. This retrospective study was approved by our Institutional Review Board (Number 2020-228).

Consent to participate: Consent to participate was waived due to the retrospective study design.

Consent for publication: Consent for publication was waived due to the retrospective study design.

\section{References}

1. Parsons DW, Jones $S$, Zhang $X$ et al (2008) An integrated genomic analysis of human glioblastoma multiforme. Science 321:1807-1812. https:// doi:10.1126/science.1164382 
2. Ichimura K, Pearson DM, Kocialkowski S et al (2009) IDH1 mutations are present in the majority of common adult gliomas but rare in primary glioblastomas. Neuro Oncol 11:341-347. https://doi:10.1215/15228517-2009-025

3. Watanabe T, Nobusawa S, Kleihues P et al (2009) IDH1 mutations are early events in the development of astrocytomas and oligodendrogliomas. Am J Pathol 174:1149-1153. https://doi:10.2353/ajpath.2009.080958

4. Louis D, Ohgaki H, Wiestler O et al (2016) WHO classification of tumours of the central nervous system, revised. IARC Press, Lyon

5. Patel SH, Poisson LM, Brat DJ et al (2017) T2-FLAIR Mismatch, an Imaging Biomarker for IDH and 1p/19q Status in Lower-grade Gliomas: A TCGA/TCIA Project. Clin Cancer Res 23:6078-6085. https://doi:10.1158/1078-0432.CCR-17-0560

6. Eckel-Passow JE, Lachance DH, Molinaro AM et al (2015) Glioma Groups Based on 1p/19q, IDH, and TERT Promoter Mutations in Tumors. N Engl J Med 372:2499-2508.

https://doi:10.1056/NEJMoa1407279

7. Kaloshi G, Benouaich-Amiel A, Diakite F et al (2007) Temozolomide for low-grade gliomas: predictive impact of 1p/19q loss on response and outcome. Neurology 68:1831-1836. https://doi:10.1212/01.wnl.0000262034.26310.a2

8. Broen MPG, Smits M, Wijnenga MMJ et al (2018) The T2-FLAIR mismatch sign as an imaging marker for non-enhancing IDH-mutant, 1p/19q-intact lower-grade glioma: a validation study. Neuro Oncol 20:1393-1399. https://doi:10.1093/neuonc/noy048

9. Warntjes JB, Dahlqvist O, Lundberg P (2007) Novel method for rapid, simultaneous T1, T2*, and proton density quantification. Magn Reson Med 57:528-537. https://doi:10.1002/mrm.21165

10. Reuss DE, Sahm F, Schrimpf D et al (2015) ATRX and IDH1-R132H immunohistochemistry with subsequent copy number analysis and IDH sequencing as a basis for an "integrated" diagnostic approach for adult astrocytoma, oligodendroglioma and glioblastoma. Acta Neuropathol 129:133146. https://doi:10.1007/s00401-014-1370-3

11. Akagi Y, Yoshimoto K, Hata N et al (2018) Reclassification of 400 consecutive glioma cases based on the revised 2016WHO classification. Brain Tumor Pathol 35:81-89. https://doi:10.1007/s10014018-0313-4

12. Fleiss LJ, Cohen J (1973) The Equivalence of Weighted Kappa and the Intraclass Correlation Coefficient as Measures of Reliability. Educ Psychol Meas 33:613-619. https://doi:10.1177/001316447303300309

13. Bartko JJ (1966) The intraclass correlation coefficient as a measure of reliability. Psychol Rep 19:311. https://doi:10.2466/pr0.1966.19.1.3

14. Bhandari AP, Liong R, Koppen J et al (2021) Noninvasive Determination of IDH and 1p19q Status of Lower-grade Gliomas Using MRI Radiomics: A Systematic Review. AJNR Am J Neuroradiol 42:94101. https://doi:10.3174/ajnr.A6875 
15. Deguchi S, Oishi T, Mitsuya K et al (2020) Clinicopathological analysis of T2-FLAIR mismatch sign in lower-grade gliomas. Sci Rep 10:10113. https://doi:10.1038/s41598-020-67244-7

16. Kinoshita M, Uchikoshi M, Sakai M et al (2020) T2-FLAIR Mismatch Sign Is Caused by Long T1 and T2 of IDH-mutant, 1p19q Non-codeleted Astrocytoma. Magn Reson Med Sci https://. doi:10.2463/mrms.bc.2019-0196

17. Johnson DR, Kaufmann TJ, Patel SH et al (2019) There is an exception to every rule-T2-FLAIR mismatch sign in gliomas. Neuroradiology 61:225-227. https://doi:10.1007/s00234-018-2148-4

18. Kickingereder P, Sahm F, Radbruch A et al (2015) IDH mutation status is associated with a distinct hypoxia/angiogenesis transcriptome signature which is non-invasively predictable with rCBV imaging in human glioma. Sci Rep 5:16238. https://doi:10.1038/srep16238

19. de la Fuente MI, Young RJ, Rubel J et al (2016) Integration of 2-hydroxyglutarate-proton magnetic resonance spectroscopy into clinical practice for disease monitoring in isocitrate dehydrogenasemutant glioma. Neuro Oncol 18:283-290. https://doi:10.1093/neuonc/nov307

20. Suh CH, Kim HS, Paik W et al (2019) False-Positive Measurement at 2-Hydroxyglutarate MR Spectroscopy in Isocitrate Dehydrogenase Wild-Type Glioblastoma: A Multifactorial Analysis. Radiology 291:752-762. https://doi:10.1148/radiol.2019182200

21. Kim H, Kim S, Lee HH et al (2016) In-Vivo Proton Magnetic Resonance Spectroscopy of 2Hydroxyglutarate in Isocitrate Dehydrogenase-Mutated Gliomas: A Technical Review for Neuroradiologists. Korean J Radiol 17:620-632. https://doi:10.3348/kjr.2016.17.5.620

\section{Figures}




\section{Inclusion criteria:}

1. consecutive patients between May 2019 and May 2020 with histopathologically and genetically established gliomas with IDH mutation

2. MRI scans that had been performed within 2 weeks prior to surgery

3. Patients had undergone synthetic MRI

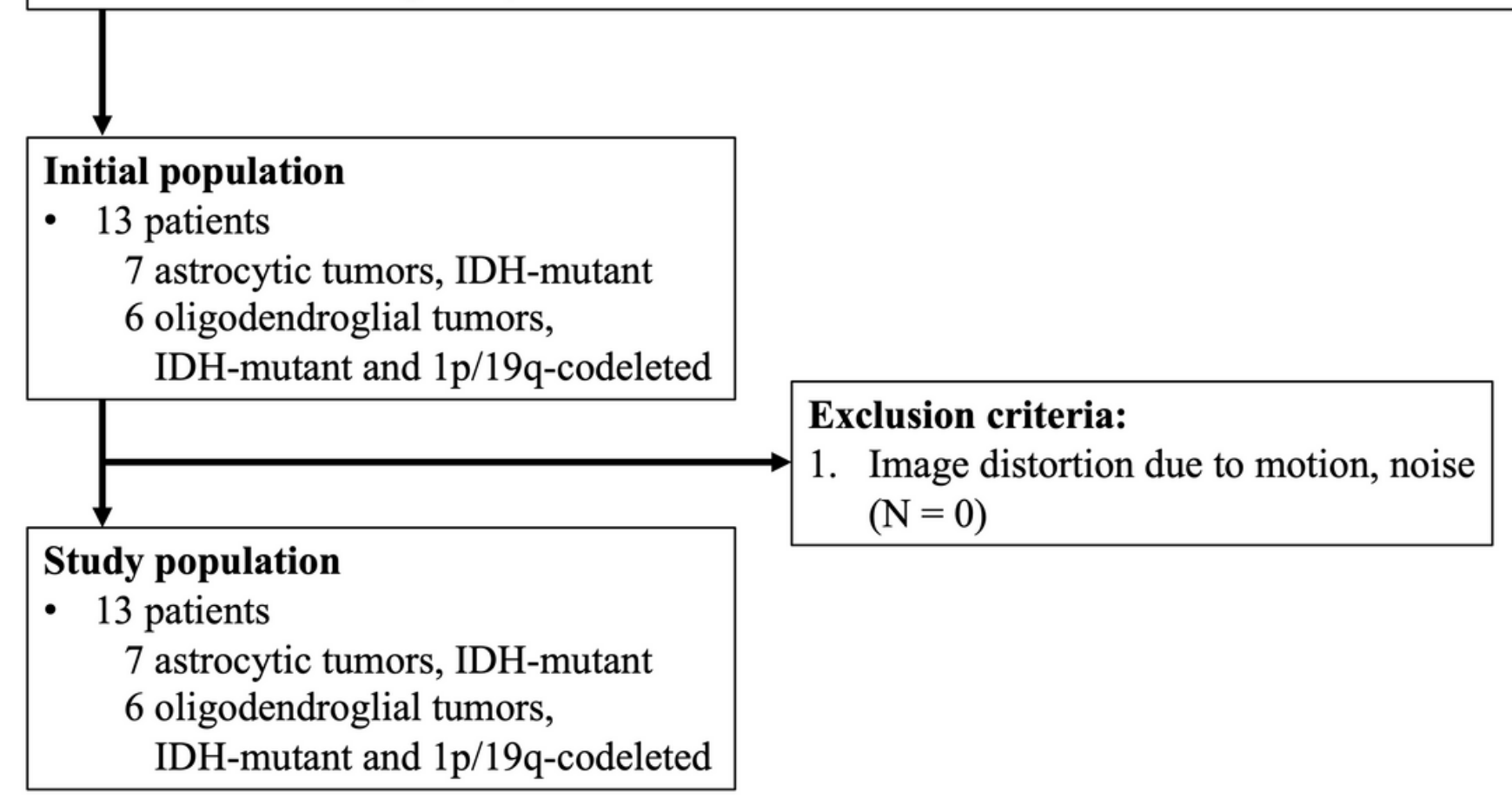

Figure 1

Patient selection flowchart IDH, isocitrate dehydrogenase 


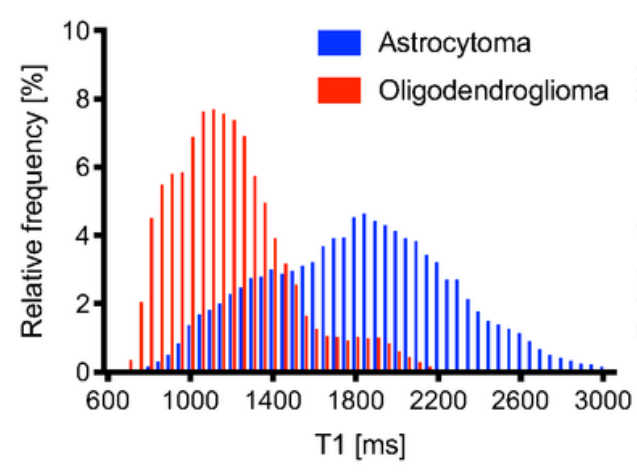

T1

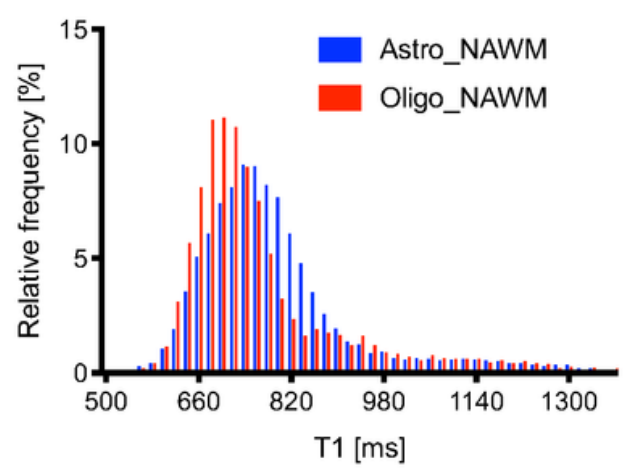

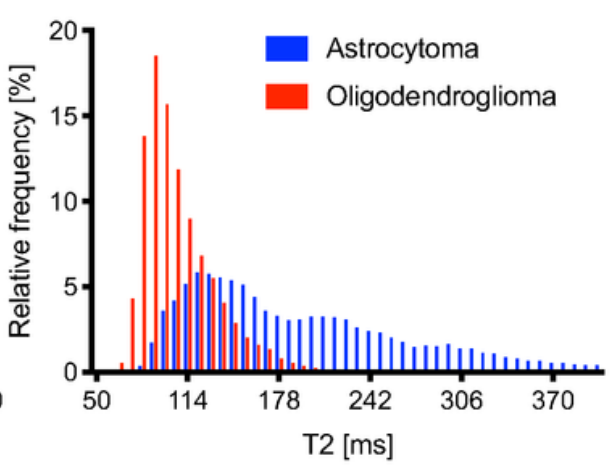

T2

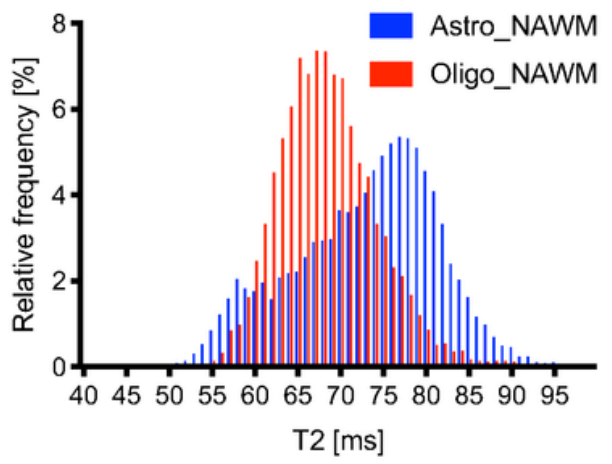

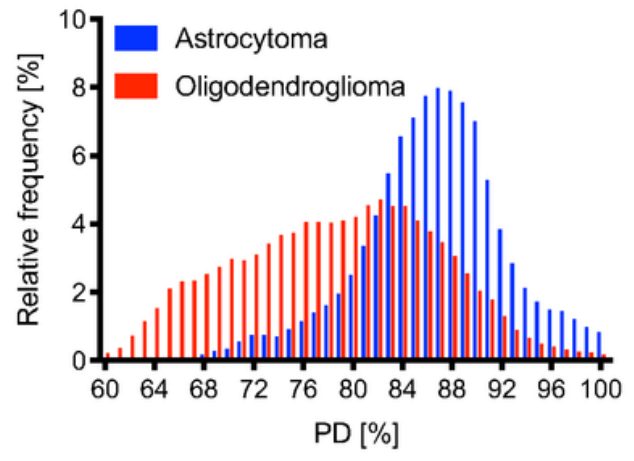

Proton Density (PD)

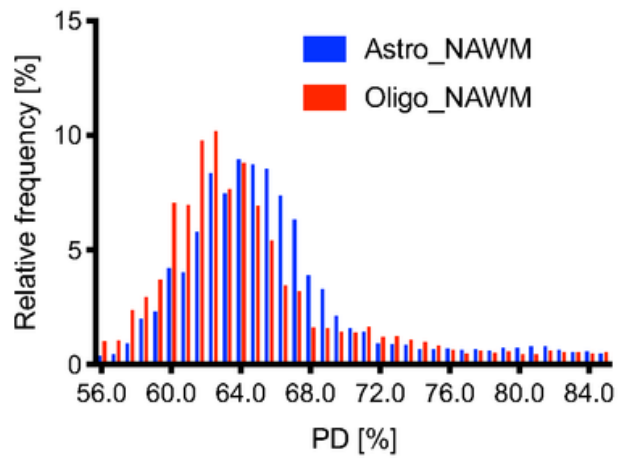

Figure 2

Histograms of $\mathrm{T} 1, \mathrm{~T} 2$, and proton density between astrocytoma, IDH-mutant and oligodendroglioma, IDHmutant and 1p/19q-codeleted All parameters (T1, T2-relaxation times, and Proton density [PD]) in astrocytoma exhibit a slight rightward shift ('+') relative to those in oligodendroglioma. There is no difference in all parameters in NAWM between the two types of gliomas. 1p/19q-codeleted, deletion of the short arm of chromosome 1 and long arm of chromosome 19; IDH, isocitrate dehydrogenase, NAWM, normal appearing white matter; PD, proton density 
T2-FLAIR mismatch sign Present

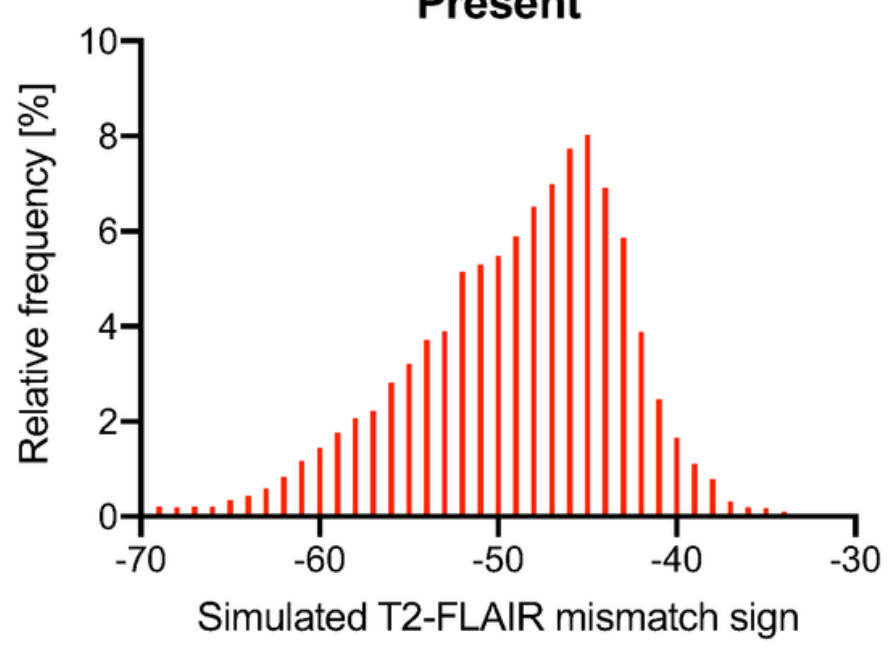

T2-FLAIR mismatch sign

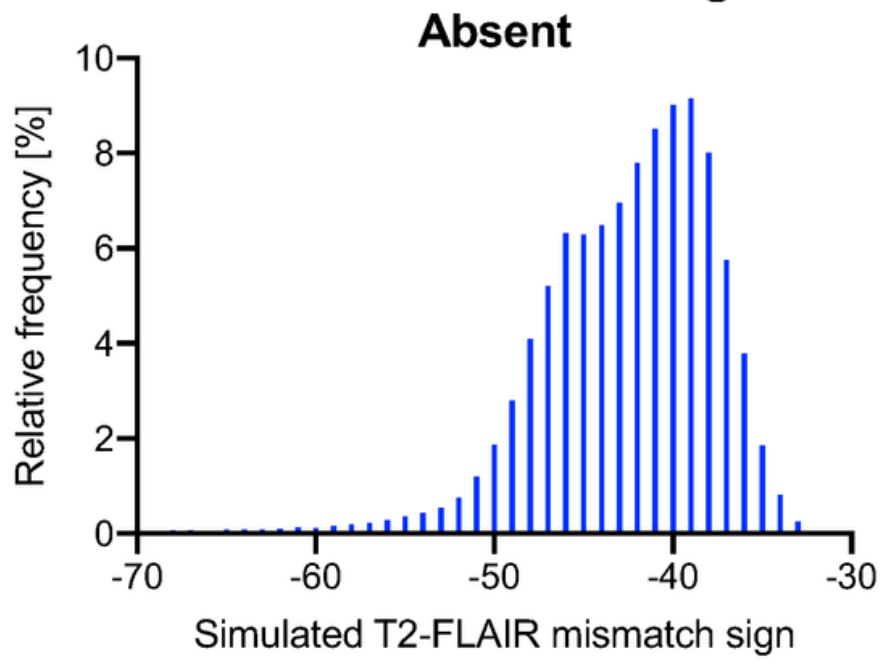

\section{Figure 3}

Simulated T2-FLAIR mismatch sign (\% change) of the present/absent T2-FLAIR mismatch cases The \% change in the present T2-FLAIR mismatch cases shows a larger \% change $(-49.3 \pm 6.5 \%)$ compared to those in the absent cases $(-43.4 \pm 7.9 \%)$. 

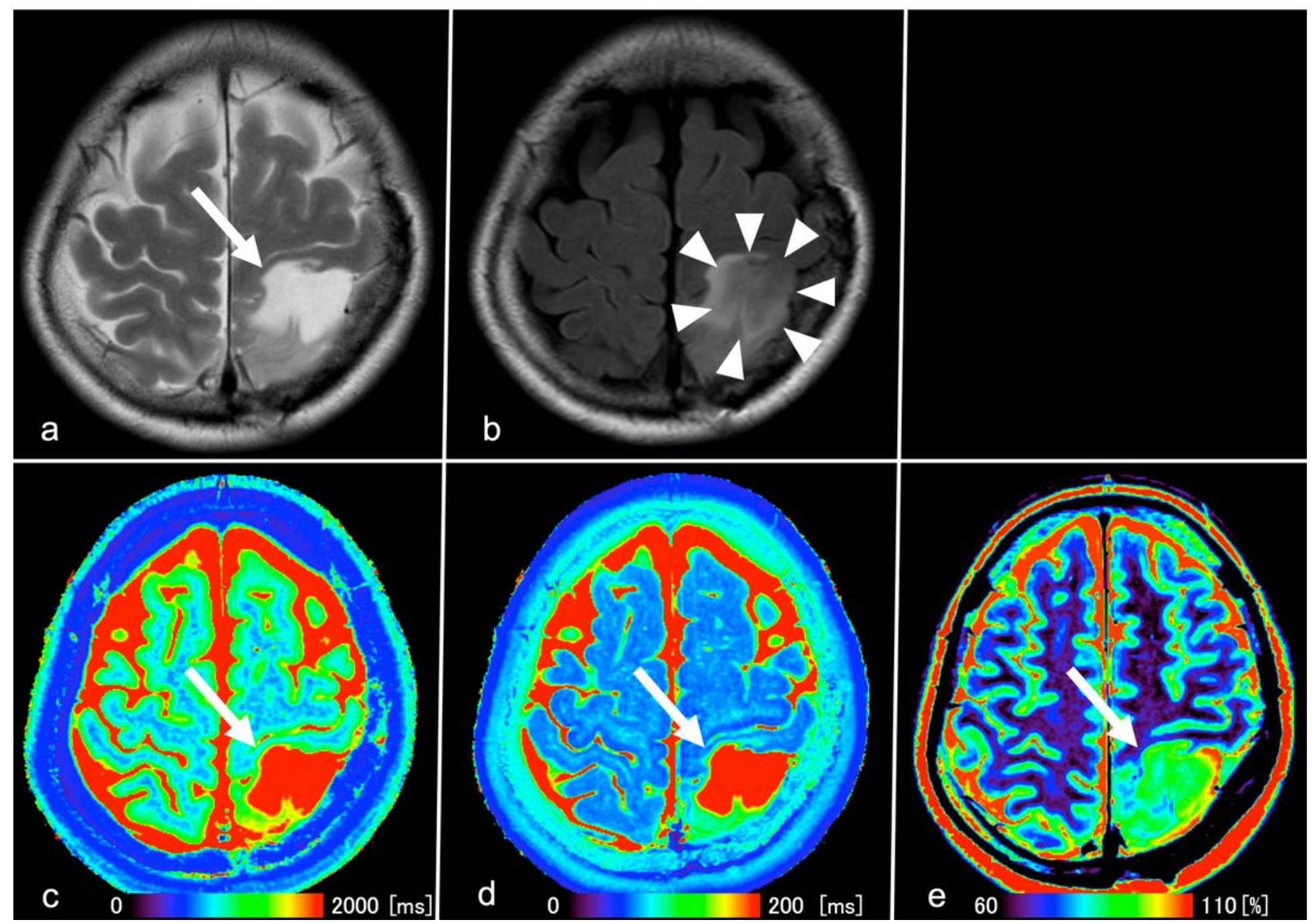

Figure 4

Images from a 49-year-old woman with diffuse astrocytoma, IDH-mutant (WHO grade II). a, T2WI shows a heterogeneous T2-prolonged mass in the left parietal lobe (arrow). b, FLAIR shows partial signal suppression, indicating a T2-FLAIR mismatch sign (arrowheads). c, d, e, T1, T2, proton density (PD) maps derived from SyMRI show T1- $\left(2436 \mathrm{~ms}^{\star}\right)$, T2- $\left(287 \mathrm{~ms}^{\star}\right)$ relaxation time prolongations, and increased PD $\left(94.9 \%^{*}\right)$ (arrows) in the tumor. IDH, isocitrate dehydrogenase; PD, proton density; SyMRI, synthetic MRI; WHO, World Health Organization *Each value expresses as mean. 


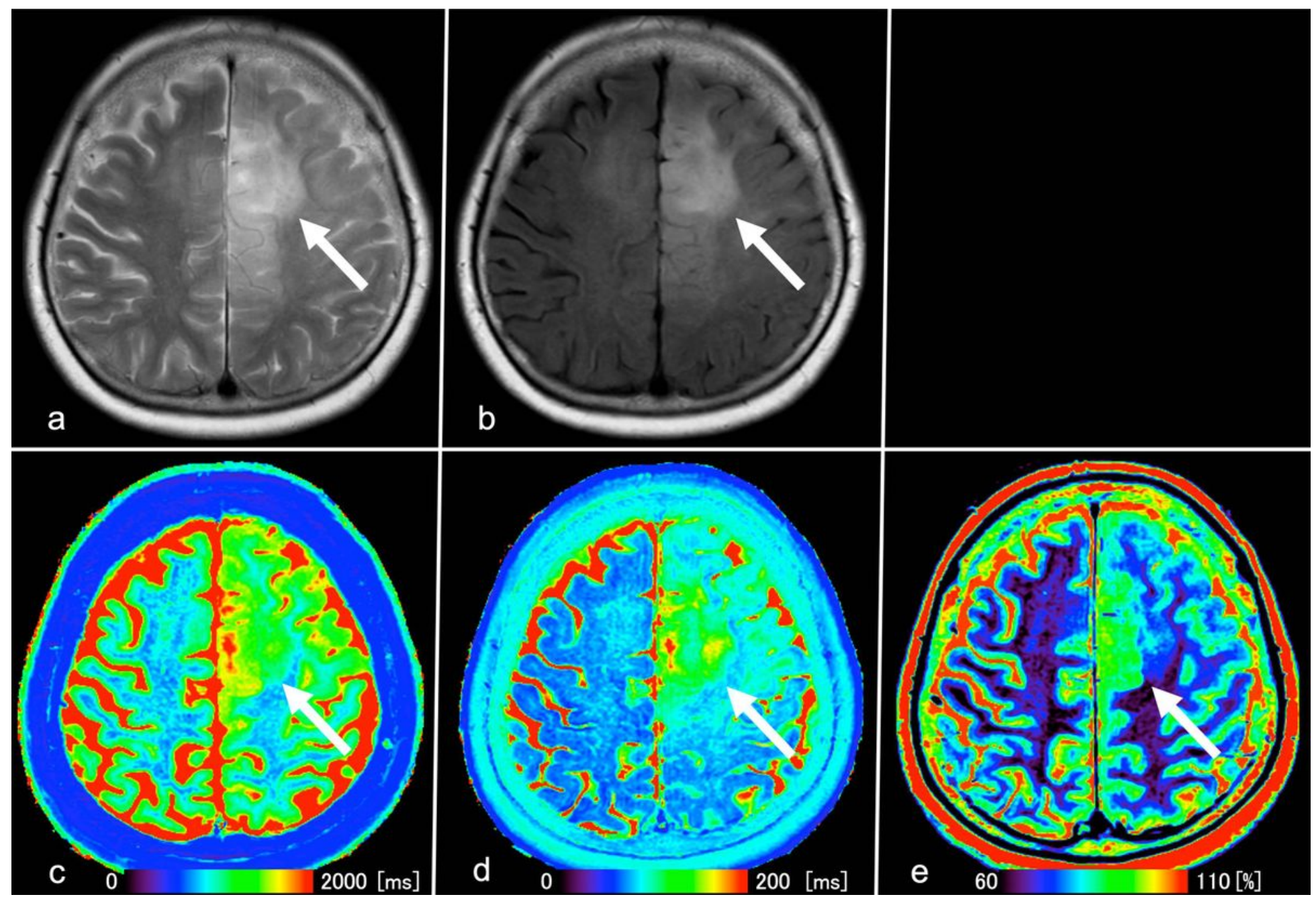

Figure 5

Images from a 63-year-old woman with oligodendroglioma, IDH-mutant and 1p/19q-codeleted (WHO grade II). a, T2WI shows a heterogeneous, poorly-circumscribed mass in the left frontoparietal lobes. b, FLAIR shows no signal suppression in the tumor, indicating no T2-FLAIR mismatch sign (arrow). c, d, e, T1, T2, proton density (PD) maps derived from SyMRI show mild T1- (1119 ms*), T2- (103 ms*) relaxation time prolongations, and mildly increased PD (76.8\%*) (arrows) in the tumor. $1 \mathrm{p} / 19 \mathrm{q}$-codeleted, deletion of the short arm of chromosome 1 and long arm of chromosome 19; IDH, isocitrate dehydrogenase; PD, proton density; SyMRI, synthetic MRI; WHO, World Health Organization *Each value expresses as mean.

\section{Supplementary Files}

This is a list of supplementary files associated with this preprint. Click to download.

- 04ESMT2FL062821.docx 